\title{
Instrumentos específicos para avaliação da qualidade de vida em obesos: uma revisão integrativa
}

Specific instruments for assessing quality of life in obese people: an integrative review

Instrumentos específicos para evaluar la calidad de vida en personas obesas: una revisión integradora

Francisca Souza Santos Dias ${ }^{1}$, Clara Cynthia Melo e Lima ${ }^{1}$, Tatiana Fróes Fernandes ${ }^{2}$, Patrícia de Sousa Fernandes Queiroz ${ }^{3}$, Hérika Maria Silveira Ruas ${ }^{4}$.

\section{RESUMO}

Objetivo: Identificar e analisar os principais instrumentos de avaliação da qualidade de vida em obesos. Métodos: Trata-se de uma revisão integrativa da literatura, cujo corpus do estudo foi formado pelas produções científicas que evidenciam os instrumentos especificamente construídos e/ou validados para medir a qualidade de vida em pacientes com obesidade. A busca orientou-se de acordo com PRISMA. Os bancos de dados PubMed, Web of Science e Scielo foram utilizados para identificar os estudos. Foram estabelecidos três blocos de termos de pesquisa: (a) população-alvo (obesidade); (b) construto de interesse (Qualidade de Vida); (c) tipo de instrumento (questionário). Resultados: Dos 5.020 artigos completos encontrados, 23 artigos descreveram desenvolvimento de um instrumento de medida e foram considerados elegíveis. Entre os estudos analisados, foram encontrados onze instrumentos específicos para a mensuração da qualidade de vida em obesidade. Ressalta-se que não foi encontrado questionário específico para obesidade desenvolvido no Brasil. Considerações finais: A utilização de um instrumento específico para a avaliação do impacto da obesidade na qualidade de vida pode ser um importante indicador de saúde, podendo subsidiar políticas públicas que almejem à melhoria da qualidade de vida.

Palavras-chave: Obesidade, Qualidade de vida, Saúde.

\begin{abstract}
Objective: To identify and analyze the main instruments for assessing the quality of life in obese people. Methods: This is an integrative literature review, whose corpus of the study was formed by scientific productions that show the instruments specifically constructed and / or validated to measure the quality of life in patients with obesity. The search was guided according to PRISMA. The PubMed, Web of Science and Scielo databases were used to identify the studies. Three blocks of search terms were established: (a) target population (obesity); (b) construct of interest (Quality of Life); (c) type of instrument (questionnaire). Results: Of the 5,020 complete articles found, 23 articles described the development of a measurement instrument and were considered eligible. Among the studies analyzed, eleven specific instruments were found to measure the quality of life in obesity. It should be noted that no specific questionnaire for obesity developed in Brazil was found. Final considerations: The use of a specific instrument for the assessment of the impact of obesity on
\end{abstract}

${ }^{1}$ Instituto Federal do Norte de Minas Gerais (IFNMG), Araçuaí - MG.

*E-mail: francisca_souzasantos@yahoo.com.br

2 Universidade Estadual de Montes Claros (UNIMONTES), Montes Claros - MG.

${ }^{3}$ Instituto Federal do Norte de Minas Gerais (IFNMG), Januária - MG.

${ }^{4}$ Instituto Federal do Norte de Minas Gerais (IFNMG), Montes Claros - MG.

SUBMETIDO EM: 3/2021

ACEITO EM: 3/2021

PUBLICADO EM: 4/2021 
quality of life can be an important health indicator and can subsidize public policies that aim to improve the quality of life.

Key words: Obesity, Quality of life, Health.

\section{RESUMEN}

Objetivo: Identificar y analizar los principales instrumentos para evaluar la calidad de vida en personas obesas. Métodos: Se trata de una revisión integradora de la literatura, cuyo corpus del estudio estuvo conformado por producciones científicas que muestran los instrumentos específicamente construidos y / o validados para medir la calidad de vida en pacientes con obesidad La búsqueda se orientó según PRISMA. Se utilizaron las bases de datos PubMed, Web of Science y Scielo para identificar los estudios. Se establecieron tres bloques de términos de búsqueda: (a) población objetivo (obesidad); (b) constructo de interés (Calidad de vida); (c) tipo de instrumento (cuestionario). Resultados: De los 5.020 artículos completos encontrados, 23 artículos describieron el desarrollo de un instrumento de medición y fueron considerados elegibles. Entre los estudios analizados, se encontraron once instrumentos específicos para medir la calidad de vida en obesidad. Cabe señalar que no se encontró ningún cuestionario específico para la obesidad desarrollado en Brasil. Consideraciones finales: El uso de un instrumento específico para la evaluación del impacto de la obesidad en la calidad de vida puede ser un indicador de salud importante y puede subsidiar políticas públicas que tengan como objetivo mejorar la calidad de vida.

Palabras clave: Obesidad, Calidad de vida, Salud.

\section{INTRODUÇÃO}

A obesidade, amplamente definida como excesso peso corporal para determinada altura, é concebida pela Organização Mundial da Saúde (OMS) como uma grande epidemia mundial, relacionada principalmente aos hábitos alimentares e à realização de atividade física. Fatores ambientais, socioculturais, fisiológicos, comportamental, genético, entre outros são evidenciados na etiologia e persistência dessa patologia complexa (HEYMSFIELD SB e WADDEN TA, 2017).

O índice de massa corporal (IMC) ainda prevalece para a definição da obesidade, mesmo não definindo exatamente a quantidade corporal de gordura ou de massa magra (MANCINI MC, 2015). O IMC é obtido por meio da razão entre o peso e o quadrado da altura do indivíduo e, conforme a classificação da OMS, o sobrepeso é determinado por valores entre 25 e $29,9 \mathrm{~kg} / \mathrm{m}^{2}$ e a obesidade por valores maiores ou iguais a $30,0 \mathrm{~kg} / \mathrm{m}^{2}$ (WHO, 1995).

De acordo com The GBD 2015 Obesity Collaborators, et al. (2017), a análise de dados de 195 países verificou-se que a prevalência da obesidade dobrou em mais de 70 países desde 1980 e mais de 600 milhões de adultos eram obesos em 2015, além de ocasionar 4 milhões de mortes em todo o mundo. No Brasil, o índice de obesos vem aumentando em conformidade com os dados mundiais. Em estudo realizado por NCD Risk Factor Collaboration (2016), que comparou o índice de massa corporal (IMC) de cerca de 20 milhões de pessoas de ambos os sexos, de diversas nacionalidades, verificou-se que o Brasil é um dos países com maior taxa de obesidade do mundo, sendo que um quinto da população brasileira é obesa.

A obesidade é atualmente uma preocupação global, tendo em vista sua associação direta com outras doenças como hipertensão arterial sistêmica, doença arterial coronariana, dislipidemia, diabetes mellitus tipo 2, acidente vascular encefálico, esteatose hepática, osteoartrite, apneia do sono, litíase biliar, disfunção cognitiva, distintos tipos de câncer, ansiedade, depressão e dor crônica (ARNOLD M, et al, 2016). Nesse contexto, cresce o interesse de pesquisadores em avaliar a qualidade de vida em obesos, uma vez que 0 impacto da obesidade não se limita apenas às questões relacionadas à saúde, mas também pode influenciar na longevidade e na qualidade de vida, podendo aumentar a probabilidade de morte e diminuir os indicadores de qualidade de vida, evidenciados, por exemplo, por dificuldades de interação social, isolamento social, baixa 
autoestima, isolamento social, estresse, depressão e consequências negativas também no desempenho laboral (GORDON PC, et al., 2011).

O conceito qualidade de vida tornou-se foco de vários estudos em diversas áreas do conhecimento, com definições distintas, dependendo do contexto de sua utilização. Atualmente as definições mais aceitas baseiam-se no princípio de que o conceito de qualidade de vida apresenta uma ampla dimensionalidade, com organização complexa e dinâmica, diferindo de pessoa para pessoa de acordo com seu ambiente ou até mesmo sendo diferente entre duas pessoas inseridas em mesmo ambiente. Diante disso, a definição de qualidade de vida é apresentada como sendo constituída por aspectos psicológicos, físicos, sociais e ambientais (SILVA SA, et al., 2021).

Considerando a ampla dimensão da saúde e sua relação com diversos aspectos da vida, a avaliação da qualidade de vida pode ser muito variável, uma vez que indivíduos com a mesma morbidade podem manifestar diferentes níveis de saúde e bem-estar físico e emocional. Assim, surgiu o termo avaliação da qualidade de vida relacionada à saúde (QVRS), baseada na percepção do indivíduo sobre o impacto da doença e seu tratamento, referindo-se a uma medição multidimensional da qualidade de vida, com avanços no número de instrumentos para medi-la (GUYATT GH, et al., 1993).

Em geral, podem ser usados dois tipos de instrumentos de avaliação da QVRS: as escalas genéricas ou específicas. As escalas "específicas" de qualidade de vida são concebidas especificamente para uma doença, neste caso a obesidade. As chamadas escalas "genéricas" de qualidade de vida são usadas e validadas em uma ampla gama de doenças crônicas. Os instrumentos específicos são bem adaptados às doenças para as quais foram concebidos e, portanto, são capazes de registrar até mesmo pequenas alterações na qualidade de vida (LE PEN C, et al., 1998).

Por outro lado, as genéricas são úteis na realização de estudos gerais sobre saúde e na comparação de diferentes estados de determinadas doenças. Considerando as limitações dos instrumentos genéricos, são recomendadas ferramentas específicas para a avaliação de aspectos específicos da QVRS de uma maneira individual, proporcionando assim uma maior capacidade de verificar os impactos da obesidade no bem-estar do indivíduo (LE PEN C, et al., 1998).

Deste modo, considerando os impactos que a obesidade pode provocar na saúde e nas atividades cotidianas e laborais do indivíduo, e a relevância da utilização de instrumentos específicos, validados, para a avaliação da qualidade de vida em situações de obesidade, este estudo tem como objetivo, realizar uma revisão integrativa da literatura especializada sobre os principais instrumentos de mensuração da qualidade de vida em indivíduos com obesidade.

\section{MÉTODOS}

Trata-se de uma revisão integrativa da literatura, cujo corpus do estudo foi formado pelas produções científicas que evidenciam os instrumentos especificamente construídos e/ou validados para medir a qualidade de vida em pacientes com obesidade. Para sistematização dessa revisão, foram seguidas seis etapas: elaboração da pergunta norteadora "Quais os instrumentos especificamente construídos e/ou validados para medir a qualidade de vida em pacientes com obesidade?"; buscas na literatura; coleta de dados; análise dos estudos incluídos; discussão dos resultados; e apresentação da revisão integrativa (MENDES KDS, et al., 2008).

A pesquisa foi orientada de acordo com Preferred Reporting Items for Systematic Review and MetaAnalysis (PRISMA). Os bancos de dados PubMed, Web of Science e Scientific Electronic Library Online (Scielo), foram utilizados para identificar os estudos. Foram selecionados artigos publicados como trabalhos em texto completo e que tivessem como objetivo o desenvolvimento e/ou avaliação das propriedades de medição de instrumentos que mensuram a qualidade de vida em obesidade.

Estudos que relataram evidências indiretas, como ensaios clínicos que medem a qualidade de vida, não foram considerados elegíveis. Os instrumentos elegíveis incluíram todos os instrumentos especificamente 
construídos e/ou validados para medir a qualidade de vida em pacientes com obesidade, que podem ser utilizados tanto em pesquisa bem como na clínica. Os artigos foram excluídos se (a) um construto diferente da qualidade de vida fosse medido, (b) instrumentos que foram desenvolvidas para crianças ou adolescentes (idade $<18$ anos), (c) Construto desenvolvido essencialmente para a avaliação da qualidade de vida de pacientes de cirurgia bariátrica ou de contorno corporal.

A busca das publicações foi realizada de junho a agosto de 2020, sendo que a principal estratégia de busca está detalhada no Quadro 1. Foram estabelecidos três blocos de termos de pesquisa: (a) populaçãoalvo (obesidade); (b) construto de interesse (Qualidade de Vida); (c) tipo de instrumento (questionário). Foi utilizado um filtro de pesquisa altamente sensível para encontrar estudos sobre propriedades instrumentos de medição, que foi desenvolvido pela Oxford University e disponível através do site do COSMIN. A pesquisa não foi limitada por ano de publicação, desenho ou idioma do estudo. A seleção dos artigos foi realizada manualmente e foram excluídos estudos que atenderam aos critérios de exclusão predefinidos. Os critérios de exclusão foram: artigos que não apresentavam relação com o objeto; relatos de experiências e de casos, monografias, dissertações, teses, resumos em anais de eventos, capítulos de livro. Artigos duplicados foram considerados uma única vez.

Quadro 1 - Estratégias de busca nas bases de dados.

\begin{tabular}{|c|c|c|c|}
\hline Base de dados & & Estratégia de busca & $\begin{array}{l}\text { № de estudos } \\
\text { encontrados }\end{array}$ \\
\hline \multirow{8}{*}{ PubMed } & $\# 1$ & $\begin{array}{l}\text { "obesity [MeSH] OR obese [All fields] OR "obesity" [All } \\
\text { fields] }\end{array}$ & 311.502 \\
\hline & \#2 & "quality of life" [MeSH] OR "quality of life" [tiab] & 285.706 \\
\hline & \#3 & "questionnaire" [MeSH] OR questionnaire" [tiab] & 1.034 .076 \\
\hline & \#4 & \#1 AND \#2 AND \#3 & 2.730 \\
\hline & $\# 5$ & Filtro & 7.510 .031 \\
\hline & \#6 & \#4 AND \#5 & 1.766 \\
\hline & \#7 & Filtro & 424.579 \\
\hline & \#8 & \#6 NOT \#7 & 1.750 \\
\hline \multirow{4}{*}{ Web of Science } & $\# 1$ & TÓPICO: (Obesity) OR TÓPICO: (obese) & 409.614 \\
\hline & \#2 & TÓPICO: (quality of life) & 510.032 \\
\hline & \#3 & TÓPICO: (questionnaire) & 631.398 \\
\hline & \#4 & \#1 AND \#2 AND \#3 & 3.130 \\
\hline \multirow{4}{*}{$\begin{array}{l}\text { Scientific } \\
\text { Electronic Library } \\
\text { Online }\end{array}$} & $\# 1$ & (obesidade) OR (obesity) OR (obeso) OR (obese) & 10.888 \\
\hline & \#2 & (qualidade de vida) OR (quality of life) & 16.780 \\
\hline & \#3 & (instrumento) OR (escala) OR (questionnaire) & 57.703 \\
\hline & \#4 & $\# 1$ AND \#2 AND \#3 & 140 \\
\hline
\end{tabular}

Fonte: Dias FSS, et al., 2021.

\section{RESULTADOS}

Dos 5.020 artigos completos encontrados, 23 artigos descreveram o desenvolvimento e validação de instrumento de medida e foram considerados elegíveis (Quadro 2). A partir da leitura dos títulos e dos resumos dos artigos por duas pesquisadoras independentes, considerando os critérios de inclusão e exclusão, foram eliminadas 4080 publicações. Duas pesquisadoras independentes detectaram 17 artigos duplicados, que foram excluídos do estudo. Alcançou-se, então, a amostra final de 23 estudos primários, que após a leitura na íntegra, os dados foram organizados para análise e interpretação por todas as pesquisadoras (Figura 1). 
Figura 1 - Fluxograma do processo de seleção dos estudos primários adaptado do Preferred Reporting Items for Systematic review and Meta-Analyses (PRISMA).

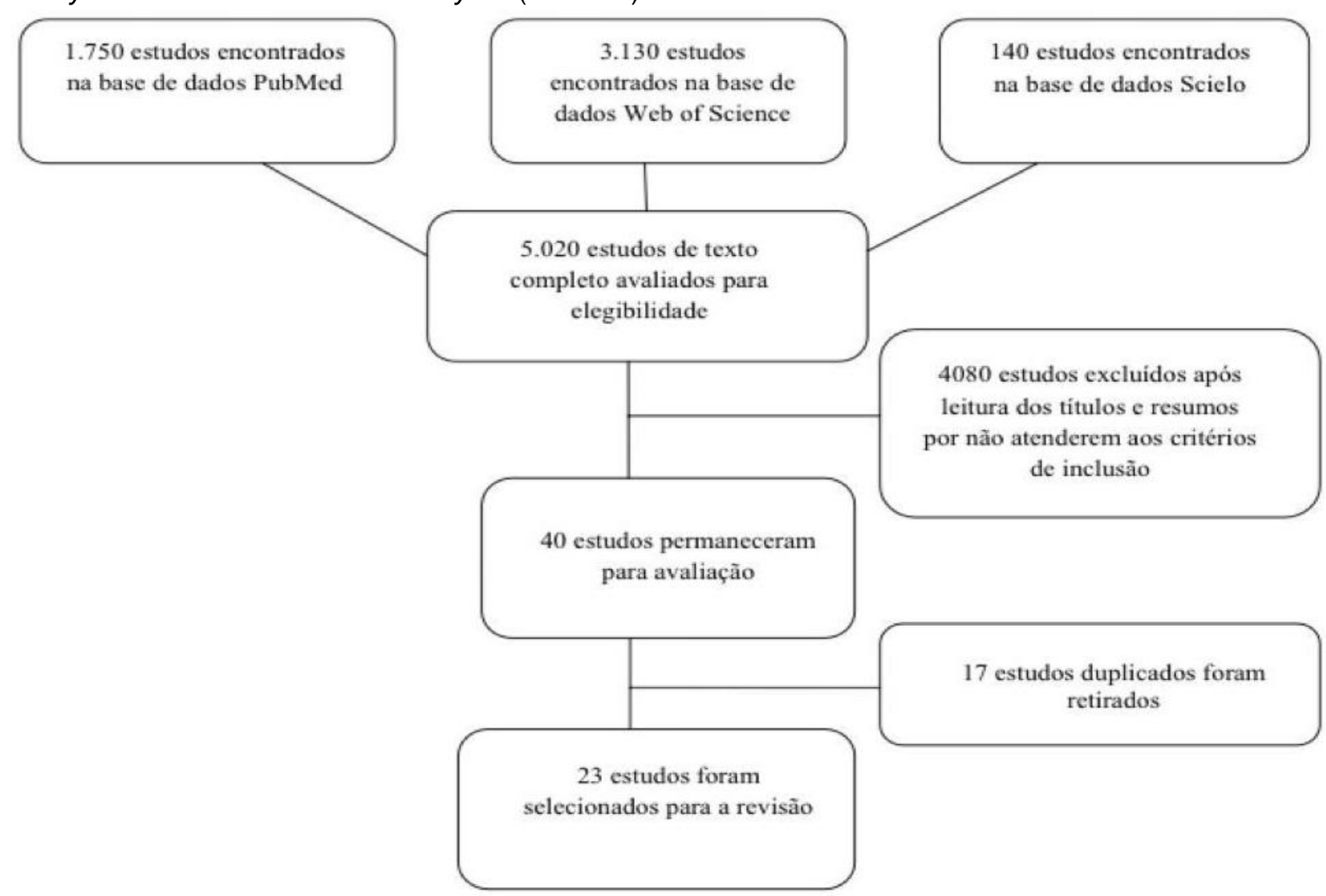

Fonte: Dias FSS, et al., 2021.

Entre os estudos analisados, foram encontrados onze instrumentos específicos para a mensuração da qualidade de vida em obesidade: The Impact of Weight on Quality of Life (IWQOL), The Impact of Weight on Quality of Life-Lite (IWQOL-Lite), The Health-related quality of life, health state preference (Lewin-TAG HSP, The Obesity Related Well-Being (ORWELL-97), The Obese Specific Quality of Life (OSQOL, The Obesity and Weight Loss Quality of Life Questionnaire (OWLQOL) e The Weight-Related Symptom Measure (WRSM), Quality Of Life, Obesity and Dietetics (QOLOD), The Obesity-related Psychosocial problems scale (OP-Scale), The Laval Questionnaire, Patient-Reported Outcomes in Obesity (PROS). Destes, cinco foram desenvolvidos em inglês (IWQOL, IWQOL-Lite, Lewin-TAG HSP, OWLQOL, WRSM), três em francês (OSQOL The Laval questionnaire), um em Italiano (ORWELL-97), um em sueco (OP-Scale) e um em norueguês (PROS). Ressalta-se que não foi encontrado questionário específico para obesidade desenvolvido no Brasil. 
Quadro 2 - Características dos diferentes estudos relacionados aos instrumentos de medição da qualidade de vida em obesos.

\begin{tabular}{|c|c|c|c|}
\hline Instrumento & Autor/Ano de publicação & Número de participantes & IMC médio \\
\hline IWQOL & Kolotkin RL, et al., 1997 & $\begin{array}{l}\text { Pacientes em tratamento para obesidade } \\
\text { Estudo 1: } 64 \\
\text { Estudo 2: } 181\end{array}$ & $\begin{array}{c}\text { Estudo 1: } \\
\text { Mulheres: } 34,6 \mathrm{~kg} / \mathrm{m}^{2} \text { para mulheres } \\
\text { Homens: } 40,4 \mathrm{~kg} / \mathrm{m}^{2} \\
\text { Estudo } 2: 38,3 \mathrm{~kg} / \mathrm{m}^{2} \\
\end{array}$ \\
\hline \multirow[t]{2}{*}{ IWQOL - Lite } & Kolotkin RL, et al., 2001 & $\begin{array}{c}\text { Indivíduos que fizeram o IWQOL anteriormente: } \\
\text { Amostras separadas usadas para escala desenvolvimento e } \\
\text { validação cruzada. }\end{array}$ & $\begin{array}{l}\text { Mulheres: } 36,6 \mathrm{~kg} / \mathrm{m}^{2} \\
\text { Homens: } 37,2 \mathrm{~kg} / \mathrm{m}^{2}\end{array}$ \\
\hline & Kolotkin RL, et al., 2002 & 494 pacientes & $27,4 \mathrm{~kg} / \mathrm{m}^{2}$ \\
\hline $\begin{array}{c}\text { IWQOL - Lite } \\
\text { (Português-Brasil) }\end{array}$ & Mariano MHA, et al., 2010 & $\begin{array}{l}\text { Amostra clínica: } 89 \text { participantes } \\
\text { Amostra da comunidade: } 156\end{array}$ & $\begin{array}{c}\text { Amostra Clínica: } 29,3 \mathrm{~kg} / \mathrm{m}^{2} \\
\text { Amostra da comunidade: } 24,4 \mathrm{~kg} / \mathrm{m}^{2}\end{array}$ \\
\hline $\begin{array}{l}\text { IWQOL - Lite } \\
\text { (Espanhol) }\end{array}$ & Andrés A, et al., 2012 & $\begin{array}{l}109 \text { participantes } \\
\text { Idade média das mulheres: } \\
\text { Média do IMC mulheres: }\end{array}$ & $\begin{array}{l}\text { Mulheres: } 47.27 \mathrm{~kg} / \mathrm{m}^{2} \\
\text { Homens: } 46.89 \mathrm{~kg} / \mathrm{m}^{2}\end{array}$ \\
\hline $\begin{array}{l}\text { Lewin-TAG HSP } \\
\text { (Inglês) }\end{array}$ & Mathias SD, et al., 1997 & $\begin{array}{c}417 \text { indivíduos divididos em três grupos: Indivíduos com } \\
\text { peso normal; Indivíduos obesos; Indivíduos mórbidos } \\
\text { obesos. }\end{array}$ & - \\
\hline $\begin{array}{l}\text { OSQOL } \\
\text { (Francês) }\end{array}$ & Le Pen C, et al., 1998 & $\begin{array}{c}\text { Grande amostra dividida em três populações: } \\
1.462 \text { indivíduos não obesos } \\
\text { 2. } 236 \text { indivíduos acima do peso } \\
\text { 3. } 155 \text { obesos }\end{array}$ & - \\
\hline OSQOL (Turco) & $\begin{array}{l}\text { Gündüzoğlu NÇ, et al., } \\
2014\end{array}$ & 170 participantes & - \\
\hline $\begin{array}{l}\text { ORWELL-97 } \\
\text { (Italiano) }\end{array}$ & Mannucci E, et al., 1999 & 147 participantes & $37,9 \mathrm{~kg} / \mathrm{m}^{2}$ \\
\hline \multirow[b]{2}{*}{$\begin{array}{l}\text { ORWELL-97 } \\
\text { (Português) }\end{array}$} & Silva IL, et al, 2008 & 198 indivíduos com obesidade & $48,76 \mathrm{~kg} / \mathrm{m}^{2}$ \\
\hline & Camolas J, et al., 2016 & $\begin{array}{c}946 \text { pessoas } \\
\text { Amostra clínica: } 188 \\
\text { Amostra comunitária: } 758 \text {. }\end{array}$ & Amostra clínica: $43,9 \mathrm{~kg} / \mathrm{m}^{2} \pm 6,45$ \\
\hline ORWELL-97 (Árabe) & Itani L, et al, 2019 & 318 participantes & - \\
\hline
\end{tabular}




\begin{tabular}{|c|c|c|c|}
\hline Instrumento & Autor/Ano de publicação & Número de participantes & IMC médio \\
\hline OP-Scale (Espanhol) & Bilbao A, et al., 2009 & 123 participantes & 49,27 \\
\hline $\begin{array}{l}\text { OP-Scale } \\
\text { (Sueco) }\end{array}$ & Karlsson J, et al., 2008 & Quatro amostras de pacientes. & $\begin{array}{l}\text { Mulher: } 23,7 \text { a } 42,7 \\
\text { Homem: } 25,1 \text { a } 41,2\end{array}$ \\
\hline OP-Scale (Koreano) & Lee YJ, et al., 2013 & 67 indivíduos & $27.90 \pm 4.49$ \\
\hline $\begin{array}{c}\text { OP-Scale } \\
\text { (Norueguês) }\end{array}$ & Aasprang A, et al., 2015 & 181 pacientes & $45 \pm 6,9$ \\
\hline $\begin{array}{l}\text { OP-Scale (Português- } \\
\text { Brasil) }\end{array}$ & Brasil AM, et al., 2017 & $\begin{array}{c}100 \text { participantes, sendo } 50 \text { pacientes obesos e } 50 \text { pacientes } \\
\text { não obesos }\end{array}$ & $\begin{array}{l}\text { Obesos: } 40,42 \\
\text { Não obesos: } 24,05\end{array}$ \\
\hline $\begin{array}{l}\text { OWLQOL e WRSM } \\
\text { (Inglês) }\end{array}$ & Niero M, et al., 2002 & $\begin{array}{c}\text { Desenvolvimento: } \\
\text { • } 68 \text { sujeitos de várias culturas } \\
\text { Validação: } \\
\text { Dados fornecidos em quatro estudos. }\end{array}$ & $\begin{array}{l}\text { Desenvolvimento: } 33,4 \mathrm{~kg} / \mathrm{m}^{2} \\
\text { Validação: } 32,9 \text { a } 37,3 \mathrm{~kg} / \mathrm{m}^{2}\end{array}$ \\
\hline The Laval (Francês) & Therrien F, et al., 2011 & 112 participantes & - \\
\hline \multirow{2}{*}{ The Laval (Italiano) } & Donini LM, et al., 2017 & 273 indivíduos & $\geq 30 \mathrm{~kg} / \mathrm{m}^{2}$ \\
\hline & Accardi R, et al., 2017 & 163 participantes & $42,8 \pm 7,5 \mathrm{~kg} / \mathrm{m}^{2}$ \\
\hline QOLOD (Francês) & Ziegler O, et al., 2005 & $\begin{array}{l}\text { Estudo 1: } 128 \text { pacientes obesos } \\
\text { Estudo 2: } 212 \text { pacientes }\end{array}$ & $\begin{array}{l}34,5 \pm 2,8 \mathrm{~kg} / \mathrm{m}^{2} \\
35,8 \pm 7,4 \mathrm{~kg} / \mathrm{m}^{2}\end{array}$ \\
\hline PROS (Norueguês) & Aasprang A, et al., 2019 & $\begin{array}{l}109 \text { participantes antes da cirurgia bariátrica } \\
95 \text { participantes após a cirurgia ( } 1 \text { a } 5 \text { anos) }\end{array}$ & $\begin{array}{l}\text { Antes da cirurgia: } 42 \mathrm{~kg} / \mathrm{m}^{2} \\
\text { Após a cirurgia: } 29 \mathrm{~kg} / \mathrm{m}^{2}\end{array}$ \\
\hline
\end{tabular}

Fonte: Dias FSS, et al., 2021 


\section{DISCUSSÃO}

\section{Avaliação da qualidade de vida em obesos}

A obesidade, principal doença crônica emergente nas últimas décadas, é um problema de saúde pública em rápido crescimento, afetando um número cada vez maior de países em todo o mundo e criando fardos financeiros e de saúde expressivos. Dentre os estudos sobre a importância de determinados fatores biológicos e psicológicos para a qualidade de vida prejudicada, a obesidade é um fator bem estabelecido (PARK S, 2017).

Situações de obesidade afetam profundamente o funcionamento físico e psicossocial, levando ao aparecimento de incapacidades, especialmente dificuldade nas atividades de vida diária, mobilidade, atividades domésticas, ao ar livre e laborais (CAPODAGLIO P e LIUZZI A, 2013). Estudo recente confirmou o impacto significativo e negativo que a obesidade tem na qualidade de vida relacionada à saúde com referência ao bem-estar físico e mental, relações sociais e fatores ambientais e econômicos (KOLOTKIN RL e ANDERSEN JR, 2017).

Qualidade de vida é um conceito multidimensional amplo que geralmente inclui avaliações subjetivas de aspectos positivos e negativos da vida. Embora a saúde física seja um dos domínios importantes da qualidade de vida geral, outros domínios, como emprego, moradia, escolas e o ambiente também são relevantes (SIMON GE, et al., 2006). A definição mais abrangente de qualidade de vida é especialmente importante para a obesidade, tendo em vista que se trata de uma doença com etiologia multifacetada e amplo impacto, influenciando distintos domínios da saúde (TAYLOR VH, et al., 2013).

A mensuração da qualidade de vida se tornou um relevante indicador de saúde, capaz de direcionar políticas públicas com vistas à melhoria da qualidade de vida, uma vez que fornece informações sobre dos impactos da condição clínica na vida do paciente (POETA LS, et al., 2010). No contexto da obesidade, o conceito de "Qualidade de Vida Relacionada à Saúde" é especialmente significativo, pois representa uma questão fundamental a ser considerada no manejo da obesidade, em que pacientes podem ter todos os domínios da QVRS podem ser gravemente afetados nesses pacientes, por outro lado podem ser satisfatórios.

Embora instrumentos genéricos, para medir a qualidade de vida relacionada à saúde, forneçam informações úteis, eles não foram projetados para medir a gama específica de problemas relacionados à saúde vivenciados por indivíduos com obesidade. Assim, um número crescente de questionários de qualidade de vida específicos para doenças estão sendo desenvolvidos nas últimas décadas (KOLOTKIN RL e ANDERSEN JR, 2017). Dentre estes, ressaltam-se, os estudos direcionados à construção e validação de instrumentos específicos para a mensuração da qualidade de vida em obesidade.

\section{Principais questionários de avaliação da atividade física e condições associadas}

Em termos gerais, qualidade de vida geralmente se refere à percepção do desempenho do paciente em pelo menos um dos quatro domínios importantes: sensação somática, função física, estado emocional e interação social9. Sendo que seis questionários incluem itens relacionados a esses quatro domínios (IWQOL, IWQOL-Lite, ORWELL-97, QOLOD, The Laval Questionnaire, PROS). As principais características dos instrumentos estão descritos a seguir:

The Impact of Weight on Quality of Life (IWQOL): esse instrumento possui 74 itens divididos em oito domínios: Saúde, Social / Interpessoal, Trabalho, Mobilidade, Auto-estima, Vida sexual, Atividades da vida diária, conforto com comida. Escala de itens: escala de 5 pontos 1 (nunca verdadeiro) a 5 (sempre verdadeiro). Pontuação total: somando a pontuação de cada item (KOLOTKIN RL, et al, 1997).

The Impact of Weight on Quality of Life-Lite (IWQOL-Lite): Esse instrumento possui 31 itens divididos em cinco domínios: Função física, Auto-estima, Vida sexual, Sofrimento público, Trabalho. Pontuações (uma pontuação total e uma para cada um dos cinco domínios) variam de 0 a 100, sendo 100 o melhor e 0 a qualidade de vida mais prejudicada. A taxa de licenciamento para disponibilização do questionário varia de acordo com o uso. Validada no Brasil (KOLOTKIN RL, et al., 2001; MARIANO MHA, et al., 2010). 
The Health-related quality of life, health state preference (Lewin-TAG HSP): Esse instrumento é específico para avaliação da qualidade de vida em pessoas com obesidade. Possui 55 itens contendo domínios globais (saúde geral, saúde comparada), domínios específicos para obesidade (sobrepeso, depressão, autoestima) e uma preferência de estado de saúde específico para obesidade $O$ estudo fornece poucas informações sobre a escala. A pontuação dos itens é específica para cada componente do questionário (escala de 0 a 100; 0 a 60; 7 a 49; 5 a 35). Disponível mediante solicitação aos autores. Não foi validado no Brasil (MATHIAS SD, et al., 1997).

The Obesity Related Well-Being (ORWELL-97): Esse instrumento possui 18 itens divididos em três domínios: sintomas (cinco itens); desconforto (sete itens); impacto (seis itens). Para cada item, a ocorrência e a relevância subjetiva são avaliadas. Escala de itens: escala Likert de 4 pontos 0 (nada ou nunca) a 3 (quase sempre) Pontuação total: somando a pontuação de cada item. Pontuações mais altas significam uma menor qualidade de vida. Disponível mediante solicitação aos autores. Não foi validado no Brasil (MANNUCCI E, et al., 1999).

The Obese Specific Quality of Life (OSQOL): Esse instrumento específico para avaliação da qualidade de vida em pessoas obesas, possui 11 itens divididos em quatro domínios: estado físico, vitalidade e desejo de fazer as coisas, relações com outras pessoas, estado psicológico. Escala de itens: escala de 5 pontos de (absolutamente verdadeiro) a (absolutamente falso). Escores mais altos significam maior qualidade de vida. Questionário de acesso público. Não foi validado no Brasil (LE PEN C, et al., 1998).

The Obesity and Weight Loss Quality of Life Questionnaire (OWLQOL) e The Weight-Related Symptom Measure (WRSM): No total, esses dois instrumentos possuem 17 itens que medem a qualidade de vida em relação ao peso, perda de peso e tratamento para perda de peso. O OWLQOL e o WRSM destinam-se a ser usados em conjunto e juntamente com outros resultados relatados pelo paciente sobre o status funcional, adesão à dieta e tratamento e satisfação com o tratamento. Escala Likert de 7 pontos: 0 (nada) a 6 (muito). Pontuação entre 0 a 100. Escores mais altos significam maior qualidade de vida. Não foi validado no Brasil (NIERO M, et al., 2002).

Quality Of Life, Obesity and Dietetics (QOLOD): Instrumento específico para avaliar a qualidade de vida relacionada à saúde (QVRS) em obesidade e seu manejo. O questionário consiste de 36 itens agrupados em cinco dimensões: impacto físico (11 itens), impacto psicossocial ( 11 itens), vida sexual (4 itens), conforto com comida (5 itens) e experiência com dieta (5 itens). As respostas para cada pergunta são expressas em uma escala de 1 a 5 (enormemente/o tempo todo (verdadeiro)) e 5 (nunca/nunca (verdadeiro)). Questionário de acesso público. Não foi validado no Brasil (ZIEGLER O, et al., 2005).

The Obesity-related Psychosocial problems scale (OP-Scale): Esse instrumento possui oito itens que medem o impacto da obesidade no funcionamento psicossocial. Desenvolvido para descrever os problemas psicossociais relacionados à obesidade na vida cotidiana. Escala de 4 pontos 0 (definitivamente não incomoda) a 3 (definitivamente incomoda). Pontuação total de 100 pontos.

Escores mais altos significam menor qualidade de vida. Questionário de livre acesso. Validado no Brasil (KARLSSON J, et al., 2008; BRASIL AM, et al., 2017).

The Laval Questionnaire: Trata-se de um questionário desenvolvido em francês, que avalia a qualidade de vida específica para obesidade mórbida a ser usado em ensaios clínicos. Esse instrumento possui 44 itens distribuídos em seis escores de domínio: sintomas, (10 itens); atividade / mobilidade ( 9 itens); higiene pessoal / vestuário (5 itens); emoções (11 itens); interações sociais ( 7 itens) e vida sexual (2 itens). Cada domínio é pontuado em uma escala Likert de 7 pontos; pontuações mais altas significam melhor qualidade de vida. Os pacientes devem indicar como a obesidade afetou sua vida nas últimas quatro semanas. Disponível mediante solicitação. Não foi validado no Brasil (THERRIEN F, et al., 2011).

Patient-Reported Outcomes in Obesity (PROS): É um questionário confiável e válido para medir a qualidade de vida específica da obesidade. Pode ser adequado para uso na prática clínica e na pesquisa. No questionário PROS, pede-se aos pacientes que relatem até que ponto consideram seu peso ou forma corporal incômodos nas seguintes áreas: atividade física, dor, discriminação, sono, vida sexual, vida social, trabalho / 
escola e autoestima. O questionário possui quatro categorias de resposta: não incomodado (0), levemente incomodado (1), moderadamente incomodado (2) e consideravelmente incomodado (3). A pontuação total deve ser usada como um indicador do grau de desconforto do paciente e calculada como um valor médio totalizando a pontuação da resposta e dividindo pelo número de respostas. No mínimo cinco perguntas devem ser respondidas. Questionário de acesso público. Não foi validado no Brasil (AASPRANG A, et al., 2019).

O crescente aumento da obesidade e a sua associação com comorbidades influem diretamente no estado de saúde físico, emocional e psicossocial, sendo frequentemente associada a consequências sociais negativas, com significativo impacto sobre o declínio da qualidade de vida. Assim, é crescente o interesse de pesquisadores em definir com precisão como medir a qualidade de vida de obesos.

Infere-se que vários instrumentos, de avaliação qualidade de vida, específicos para obesidade foram introduzidos durante a última década. Esses instrumentos variam amplamente em seu enfoque e perspectiva sobre qualidade de vida relacionada à saúde especialmente no que diz respeito à sua abordagem conceitual e desenho geral de medição. Um desafio para os profissionais de pesquisadores é selecionar a ferramenta mais adequada para sua população e desenho de estudo específicos. Uma vez que a pesquisa sobre os impactos da obesidade ainda carece de experiência sobre as avaliações de qualidade de vida, assim uma consideração mais cuidadosa das propriedades de cada instrumento torna-se relevante. Portanto, para uma avaliação pertinente os instrumentos devem ser bem estruturados (KARLSSON J, et al., 2003).

A utilização de um instrumento específico para a avaliação do impacto da obesidade na qualidade de vida pode ser um importante indicador de saúde, por fornecer informações a respeito da influência da condição clínica na vida do paciente, podendo subsidiar políticas públicas que almejem à melhoria da qualidade de vida.

\section{CONSIDERAÇÕES FINAIS}

Nessa revisão integrativa foram identificados onze questionários específicos validados para a medição da qualidade de vida em obesos, sendo que não foi encontrado nenhum instrumento desenvolvido no Brasil. A partir dos resultados obtidos com esse estudo, infere-se que a utilização de instrumentos específicos para avaliação da qualidade de vida em situações de obesidade pode possibilitar uma maior compreensão desse problema de saúde pública, permitindo uma análise do impacto negativo da qualidade de vida nas atividades de vida diária dos obesos.

\section{REFERÊNCIAS}

1. AASPRANG A, et al. Patient-reported quality of life with obesity - development of a new measurement scale. Tidsskr Nor Laegeforen. 2019;139(11).

2. AASPRANG A, et al. Psychosocial functioning before and after surgical treatment for morbid obesity: reliability and validation of the Norwegian version of obesity-related problem scale. 2015, PeerJ. 3:e1275;

3. ACCARDI R, et al. Italian Version of the Laval Questionnaire:Validity and Reliability. Bariatric Surgical Practice and Patient Care, 2017; 12(3).

4. ANDRÉS A, et al. Psychometric Evaluation of the IWQOL-Lite (Spanish Version) When Applied to a Sample of Obese Patients Awaiting Bariatric Surgery. OBES SURG (2012) 22:802-809.

5. ARNOLD M, et al. Obesity and cancer: an update of the global impact. Cancer Epidemiol. 2016;41:8-15.

6. BILBAO A, et al. Validation of the Spanish Translation of the Questionnaire for the Obesity-Related Problems Scale. OBES SURG (2009) 19:1393-1400.

7. BRASIL AM, et al. Adaptação transcultural e validação para o Brasil da Obesity-related Problems Scale. Einstein. 2017;15(3):327-33

8. CAMOLAS J, et al. Assessing quality of life in severe obesity: development and psychometric properties of the ORWELL-R. Eating and Weight Disorders - Studies on Anorexia, Bulimia and Obesity. 21: 277-288. 2016.

9. CAPODAGLIO P, LIUZZI A. Obesity: a disabling disease or a condition favoring disability? Eur J Phys Rehabil Med. 2013;49:395-8

10. DONINI LM, et al. Validation of the Italian version of the Laval questionnaire: health-related quality of life in subjects with obesity. Health and Quality of Life Outcomes (2017) 15:101. 
11. GORDON PC, et al. Aspectos do acompanhamento psiquiátrico de pacientes obesos sob tratamento bariátrico: revisão. Rev Psiquiatr Clin. 2011;38(4):148-54.

12. GÜNDÜZOĞLU NÇ, et al. Obezlere Özgü Yaşam Kalitesi Ölçeğinin geçerlilik ve güvenirliğinin incelenmesi. Anadolu Psikiyatri Derg 2014; 15:63-68.

13. GUYATT GH, et al. Measuring health-related quality of life. Ann Intern Med 1993; 118(8):622-629.

14. HEYMSFIELD SB, WADDEN TA. Mechanisms, pathophysiology, and management of obesity. N Engl J Med. 2017. $376: 1492$.

15. ITANI L, et al. Validation of an Arabic Version of the Obesity-Related Wellbeing (ORWELL 97) Questionnaire in Adults with Obesity. Current Diabetes Reviews. 15. 2019.

16. KARLSSON J, et al. Psychosocial functioning in the obese before and after weight reduction: construct validity and responsiveness of the Obesity-related Problems scale. International Journal of Obesity (2003) 27, 617-630.

17. KOLOTKIN RL, et al. Construct validity of the Impact of Weight on Quality of Life Questionnaire. Obes Res 1997; 5: 434-441.

18. KOLOTKIN RL, et al. Development of a brief measure to assess quality of life in obesity. Obes Res 2001; 9: 102-111.

19. KOLOTKIN RL, ANDERSEN JR. A systematic review of reviews: exploring the relationship between obesity, weight loss and health-related quality of life. Clin Obes, 2017; 5: 273-89.

20. KOLOTKIN RL, CROSBY RD. Psychometric evaluation of the impact of weight on quality of life-lite questionnaire (IWQOL-lite) in a community sample. Qual Life Res 2002; 11: 157-171.

21. LEE, YJ, et al. OP scale: QoL questionnaire for obese patients. J Korean Surg Soc. 2013;84:140-153.

22. LE PEN C, et al. 'Specific' scale compared with 'generic' scale: a double measurement of the quality of life in a French community sample of obese subjects. J Epidemiol Community Health 1998; 52: 445-450.

23. MANCINI MC. Tratado de obesidade. 2 ed. Rio de Janeiro: Guanabara Koogan, 2015

24. MANNUCCI E, et al. Quality of life and overweight: the obesity related well-being (Orwell 97) questionnaire. Addict Behav 1999; 24: 345-357.

25. MARIANO MHA, et al. Psychometric Evaluation of a Brazilian Version of the Impact of Weight on Quality of Life (IWQOL-Lite) Instrument. European Eating Disorders Review. Num. 18. 2010. p.58-66.

26. MATHIAS SD, et al. Assessing health-related quality-of-life and health state preference in persons with obesity: a validation study. Qual Life Res 1997; 6: 311-322.

27. MENDES KDS, et al. Revisão Integrativa: método de pesquisa para a incorporação de evidências na saúde e na enfermagem. Texto Contexto Enferm., 2008; 17(4): 758-764.

28. NCD Risk Factor Collaboration. Trends in adult body-mass index in 200 countries from 1975 to 2014: a pooled analysis of 1698 population-based measurement studies with $19 \cdot 2$ million participants. The Lancet. 2016. 387, 1377-1396.

29. NIERO M, et al. A new approach to multicultural item generation in the development of two obesity-specific measures: the Obesity and Weight Loss Quality of Life (OWLQOL) questionnaire and the Weight-Related Symptom Measure (WRSM). Clin Ther 2002; 24: 690-700.

30. PARK S. Pathways linking obesity to health-related quality of life. Qual Life Res (2017) 26:2209-2218.

31. POETA LS, et al. Qualidade de vida relacionada à saúde de crianças obesas. Rev. Assoc. Med. Bras., $2010 ; 56$ (2): 168-172.

32. SILVA SA, et al. Qualidade de vida e aspectos sociodemográficos de doentes renais crônicos. Revista Eletrônica Acervo Saúde, 2021, 3(2): 5475.

33. SIMON GE, et al. Association between obesity and psychiatric disorders in the US adult population. Archives of General Psychiatry, 2006; 63(7): 824-830.

34. SILVA IL, et al. Contributo para a adaptação para a população portuguesa de uma escala de avaliação da qualidade de vida específica para doentes com obesidade: a ORWELL-97. Psic., Saúde \& Doenças [online], 2008; 9(1): 29-48.

35. TAYLOR VH, et al. The impact of obesity on quality of life. Best Practice \& Research Clinical Endocrinology \& Metabolism, 2013; 27: 139-146.

36. TESSIER A, et al. Content identification of the IWQOL-Lite with the International Classification of Functioning, Disability and Health. Qual Life Res, 2011; 20: 467-477.

37. The GBD 2015 Obesity Collaborators, et al. Health effects of overweight and obesity in 195 countries over 25 years. N Engl J Med, 2017; 377:13-27.

38. THERRIEN F, et al. The laval questionnaire: a new instrument to measure quality of life in morbid obesity. Health Qual Life Outcomes, 2011; 9: 66.

39. WORLD HEALTH ORGANIZATION (WHO). Physical status: The use and interpretation of anthropometry. Technical Report Series, no 854. Genebra: World Health Organization; 1995.

40. ZIEGLER O, et al. Health related quality of life, obesity and dietetics. Diabetes Metab, 2005; 31: $273-283$. 